\title{
Mating flights, number of spermatozoa, sperm transfer and degree of polyandry in Apis koschevnikovi (Buttel-Reepen, 1906)
}

\author{
G Koeniger 1, N Koeniger 1, S Tingek ${ }^{2}$ \\ 1 Institut für Bienenkunde, Polytechnische Gesellschaft, FB Biologie an der JW Goethe-Universität, \\ K-v-Frisch-Weg 2, 61440 Oberursel, Germany; \\ 2 Agricultural Research Station Tenom, PO Box 197, 89908 Tenom, Sabah, Malaysia
}

\begin{abstract}
Summary - In Apis koschevnikovi drones and queens leave the colony for mating. Drones left the hive between $16.45 \mathrm{~h}$ and $18.30 \mathrm{~h}$. The flight duration of drones varied between less than $1 \mathrm{~min}$ and $27 \mathrm{~min}$. Drone flights longer than 10 min occurred mainly at the end of the flight time between $17.45 \mathrm{~h}$ and $18.15 \mathrm{~h}$. Queens left the hive between $17.00 \mathrm{~h}$ and $18.15 \mathrm{~h}$. Here, too, mating flights (return with a mating sign) occurred at the end of the flight period $(17.53 \mathrm{~h}$ and $18.29 \mathrm{~h})$. The flight duration of these flights was $19 \pm 5 \mathrm{~min}$. The oviducts of queens with a mating sign contained 12-21 mio spermatozoa after one mating flight. Since a mature drone has $1.7 \pm 0.16$ mio spermatozoa, this indicated multiple mating with 7-12 drones during one flight. The spermathecae contained 2-3 mio spermatozoa. The mating sign had no chitin plates. A comparison of reproductive data resulted in close similarities between $A$ cerana and $A$ koschevnikovi.
\end{abstract}

mating flight / number of spermatozoa / polyandry / A koschevnikovi

\section{INTRODUCTION}

The cavity nesting 'red honeybee' was rediscovered recently in Sabah, Borneo (Tingek et al, 1988; Mathew and Mathew, 1990). Maa described this species in 1956 and assigned the name Apis vechti. Ruttner et al (1989) reported that a red-colored bee species of Borneo had previously been described by Buttel-Reepen in 1906, who had named the species Apis koschernikovi which is the valid name because of its priority.

Generally, A koschevnikovi is similar to the sympatric $A$ cerana but larger and more reddish in color. Reproductive isolation from the 3 other sympatric Apis species of Borneo was demonstrated by the different drone flight time (Koeniger et al, 1988). The morphology of the endophallus of $A$ koschevnikovi drones is distinctly different from the Apis cerana endophallus (Tingek et al, 1988; Koeniger et al, 1991). Furthermore, morphometric studies on worker bees of $A$ koschevnikovi resulted in a significant separation from the other 2 cavity-nesting species by multivariate analysis (Rinderer et al, 1989; Ruttner et al, 1989).

The mating behavior of the European honeybee has been the focus of scientific 
interest for many years starting with the first analysis of the queen's mating flight by Janscha (1775). A concise review was published by Ruttner (1985). Further details of mating behavior in A cerana have also been reported (Ruttner and Maul, 1969; Ruttner et al, 1972, 1973; Ruttner, 1973; Woyke, 1975; Ruttner and Maul, 1983; Punchihewa et al, 1990; Punchihewa, 1992; Yoshida and Saito, 1990; Yoshida et al, 1994; Fujiwara et al, 1994). Thus, there is a great deal of information available on the reproductive behavior of the 2 cavity nesting honeybees $A$ mellifera and $A$ cerana, while even basic data on the reproductive biology of $A$ koschevnikovi are lacking. We report here on flight behavior of queens and drones, their weight, on the number of spermatozoa in drones, in the oviducts of queens returning from mating, and in the spermatheca after the start of oviposition.

\section{MATERIALS AND METHODS}

All experiments were carried out at the Agricultural Research Station in Tenom, Sabah, Borneo, Malaysia. A koschevnikovicolonies were kept in movable frame hives for several years. These colonies originate from natural swarms, which were captured with trap hives in the station or in the surrounding area.

To determine body weight, workers, queens and drones were collected from combs and confined to small plastic vials. They were immediately weighed on a digital balance (scale $0.1 \mathrm{mg}$ ).

For our experiments, we introduced sealed queen cells into small queenless colonies ( $2000-3000$ bees). We checked the colonies daily and the freshly emerged queens were marked with numbered tags for individual recognition.

An entrance channel was fitted to each hive with a glass cover on top and a queen excluder screen at the front (Ruttner, 1954). The workers passed easily through the excluder (gap $4.0 \mathrm{~mm}$ ) while the A koschevnikovi queens could not because they were much larger. The queens were released for flight as soon as they appeared in the channel. Upon return queens were found on the excluder and carefully inspected for a mating sign. The times of departure and return were recorded. We observed the flights of 7 queens.

Drone flights were observed at the hive entrance. The duration of drone flights was determined using individually marked drones (different color bars, Edding lac marker 750). Three colonies with drones were watched for drone flight time.

The number of spermatozoa was determined by dissecting queens and drones (Mackensen and Ruttner, 1976). Spermatozoa collected from the vesicula seminales or from the spermatheca were dispersed in $0.5 \mathrm{ml} 1 \% \mathrm{NaCl}$. They were then further diluted and killed by adding $4.5 \mathrm{ml}$ distilled water before counting them in a hemocytometer (Fuchs-Rosenthal). The spermatozoa collected from the oviducts were dispersed in $1 \mathrm{ml}$ $1 \% \mathrm{NaCl}$ and then diluted in $19 \mathrm{ml}$ distilled water. Five queens were dissected after mating: 3 were captured when returning with the first mating sign; 1 was dissected when returning with the second mating sign; and 1 was dissected after the start of oviposition.

\section{RESULTS}

\section{Weights}

The two egglaying queens had weights of $179 \mathrm{mg}$ and $171 \mathrm{mg}$, while the 3 queens returning from mating flights weighed 145 $\mathrm{mg}, 150 \mathrm{mg}$ and $153 \mathrm{mg}$ (table I). Drones had an average weight of $101.2 \pm 4.1 \mathrm{mg}$ $(n=20)$ and workers $72.1 \pm 7.7 \mathrm{mg}(n=$ $20)$. The number of ovarioles (both ovaries) in queens was $137.4 \pm 3.9(n=5)$. The diameter of the spermatheca of 7 queens was $d=0.74 \pm 0.03 \mathrm{~mm}$ and the volumes were about $0.25 \mathrm{~mm}^{3}$.

\section{Queen flights}

The flight activity of queens began at 17.10 and the last departure was at $18.13 \mathrm{~h}$ (fig 1 ), the median time was $17.46 \mathrm{~h} \pm 15 \mathrm{~min}$. The flights before $17.53 \mathrm{~h}$ were short flights 
for orientation, the average duration was $1.9 \pm 1.4 \mathrm{~min}(n=25$, minimum $1 \mathrm{~min}, \max -$ imum $7 \mathrm{~min}$ ). In the case of successful matings (return with a mating sign, fig 3 ) queens were flying for an average of $19 \pm 5 \mathrm{~min}(n=$ 9 , minimum $12 \mathrm{~min}$, maximum $27 \mathrm{~min}$, fig 2). All queens flying longer than $12 \mathrm{~min}$ returned with a mating sign. The first successful mating flight started at $17.53 \mathrm{~h}$, the last at $18.03 \mathrm{~h}$. The last queen returned at $18.29 \mathrm{~h}$. Thus matings occurred in a short period of only $36 \mathrm{~min}$. The median starting time for successful matings was $17.59 \pm 3.6$ $(n=9)$.

Six queens performed their orientation flights at the age of 4 and $5 \mathrm{~d}$ and their mating flights at the age of 5 and $6 \mathrm{~d}$. Three queens were dissected after the first mating and 3 were allowed to enter the hive. These queens undertook 1 or 2 more orientation flights before they left for a second mating flight. On average, the queens made 3.5 orientation flights. One queen started to fly only at the age of $7 \mathrm{~d}$ and disappeared at the age of $11 \mathrm{~d}$ after 10 orientation flights.

\section{Drone flights}

The drones started flying at $16.45 \mathrm{~h}$ in the afternoon and ceased at $18.30 \mathrm{~h}$ (fig 1). Most starts (85\%) occurred between 17.15 $\mathrm{h}$ and $18.00 \mathrm{~h}$. The duration of drone flights differed from less than $1 \mathrm{~min}$ to $27 \mathrm{~min}$ (fig 2). There were 2 maxima: the first in the range between 1 and $5 \mathrm{~min}$ and the second between 10 and $20 \mathrm{~min}$. The flights were classified like queen flights (Drescher, 1968) as mating flights (longer than $10 \mathrm{~min}$ ) and as short orientation and cleansing flights (1-10 min). Most mating flights (93\%) occurred during $1 \mathrm{~h}(17.30 \mathrm{~h}-18.30 \mathrm{~h})$, while short flights occurred during the whole flight time of $1 \mathrm{~h} 45 \mathrm{~min}$. The median time for all departures was at $17.45 \mathrm{~h} \pm 11 \mathrm{~min}$, the median time for mating flights was at $17.53 \mathrm{~h} \pm 10$ min. Using all the flights, the average flight duration was calculated as $12.3 \pm 8 \mathrm{~min}$ ( $n$ $=66$ ). Excluding the orientation flights the average was $16 \pm 6 \min (n=48)$.

\section{Mating sign}

The mating sign consisted of a thick white 'thread' of mucus, which was folded in a zigzag line within a thin transparent cover. At its surface the orange-colored cornual secretion was attached partly overlapping at the distal end (figs 3,4 ). In contrast to $A$ mellifera there were no chitin plates.

\section{Number of spermatozoa}

\section{Drones}

The vesiculae seminales of mature drones contained $1.7 \pm 0.16$ mio spermatozoa ( $n$ $=20$; table II). In the ejaculate of artificially everted drones, there were between $1.2 \pm$ 0.13 mio $(n=5)$.

\section{Queens}

The oviducts of 3 queens returning from mating flight contained $16.3 \mathrm{mio}, 19.0 \mathrm{mio}$ and 21.1 mio spermatozoa. One queen, which undertook a second mating flight the following day, had 12 mio spermatozoa in her oviducts and 2 mio in her spermatheca.

The spermatheca of a queen that started oviposition after 2 mating flights contained 3.0 mio spermatozoa. The number of spermatozoa of egglaying queens of unknown age was 1.4 mio, 1.6 mio and 2.5 mio.

\section{Number of matings of 4 queens}

Three queens had between 16.3 and 21.1 mio spermatozoa in the oviducts when they returned with a mating sign. Thus they 
mated with 10-12 drones during one flight. One queen received 12 mio spermatozoa on the second flight while 2 mio spermatozoa were already present in the spermatheca. This queen also mated more than 10 times. Thus mating with more than 10 drones seems to be normal for $A$ koschevnikovi queens.

\section{DISCUSSION}

Like in the other 2 cavity nesting species, drones and queens leave the colony for mating. The drone flight started 15 min earlier and lasted 15 min longer than the queen flight, while the median time for both was about the same (17.45 $\mathrm{h}$ and $17.46 \mathrm{~h}$, respectively). In regard to the flight duration, 2 classes were separated: flights shorter than $10 \mathrm{~min}$ (orientation flights) and longer than 10 min (mating flights), as described by Ruttner (1985) for queens and Drescher (1968) for drones of $A$ mellifera. The mating flights of drones were performed during a period of $1 \mathrm{~h}$, the queen mating flights occurred within 30 min. However, the short period for queen mating flights reported here may be partially due to the small number of flights.

The duration of the queen mating flights varied between 12 and $27 \mathrm{~min}$. This is similar to data of $A$ mellifera queens (Ruttner, 1985) and A cerana indica Fabricius 1793 queens in India (Adlakha, 1971: 15-29 min; Woyke, 1975: average of $27 \mathrm{~min}$ ). However, the mating flights were considerably longer than in Sri Lanka where $A c$ indica queens mated in 7-12 $\min (n=28$, Punchihewa, 1992). These differences may be caused by the availability of drones within the vicinity of young queens. Furthermore, environmental conditions may have some influence.

The duration of orientation flights of drones was within the range of $A$ mellifera and $A$ cerana, while the mating flights of drones (10-20 $\mathrm{min}$ ) were shorter than in $A$ mellifera (20-30 min, Drescher, 1968; Berg, 1992), but longer than in $A c$ indica in Sri Lanka (12 min, Punchihewa, 1992).

The morphology of the mating sign differs clearly from $A$ mellifera because there are no chitin plates. Furthermore, the sign is more elongated and the orange-colored cornual secretion (Koeniger et al, 1990) converges at the distal end. This is very similar to that of $A$ cerana (Ruttner et al, 1973; Woyke, 1975; Punchihewa, 1992).

During the mating flight the $A$ koschevnikovi queen collects sperm of 7-12 drones in the oviducts. This represents a large surplus of spermatozoa, less than $20 \%$ of it is stored in the spermatheca. Queens of all 3 cavity nesting species seem to mate with 10-12 drones on average ( $A C$ indica: Woyke, 1975; Punchihewa, 1992; European A mellifera: Woyke, 1960; Cornuet et al, 1986; Haberl and Moritz, 1994). In South America, 17 matings were estimated (Adams et al, 1977), and 30 matings were calculated for an $A$ cerana queen from Pakistan flying in Germany (Ruttner et al, 1973). According to Ruttner (1988), this queen belonged to the northern population $A$ cerana cerana Fabricius 1793.

The number of spermatozoa in the vesicula seminales in the 2 South Asian cavity nesting species are significantly different but in both the amount is much smaller than in $A$ mellifera (table II). In A c cerana the drone is larger (Ruttner, 1988) and the number of spermatozoa with 1.5 mio is higher (Ruttner and Maul, 1969, 1983) than in AC indica ( 1 mio). This seems to follow the general rule that larger drones have more spermatozoa. On the other hand, $A$ mellifera drones have about twice the weight of $A$ koschevnikovi drones but 5 times more spermatozoa. A koschevnikovi drones weigh $17 \%$ more than $A c$ indica drones and have about $40 \%$ more spermatozoa (tables I, II). In A mellifera small drones (originating from worker cells) have a similar amount of spermatozoa as drones of full size (Berg, 1992). 
A florea drones have about the same weight as A c indica drones (Koeniger et al, 1994) but only $40 \%$ of their spermatozoa. These data demonstrate that the amount of spermatozoa produced by a drone depends on both size and species-specific characteristics. For example, in $A$ florea the direct sperm transfer into the spermatheca (Koeniger et al, 1989) must be taken into consideration.

A koschevnikovi is considerably larger than sympatric $A$ cerana. The weight of all colony members is about a fifth higher. However, the weight relationship of workers to sexuals is about the same in both. The relationship between worker and drone is 1:1.4 and the queen has about twice the weight of a worker (1:2.1). This is different in $\mathrm{A} \mathrm{me/}$ lifera where both sexuals have more than twice the weight of a worker (1:2.4:2.3; Koeniger et al, 1994).

The number of ovarioles is about 140 in both sympatric species $A$ cerana and $A$ koschevnikovi, though $A$ koschevnikovi queens are about $25 \mathrm{mg}$ heavier. The spermathecae have about the same size, but in average the spermathecae of $A$ koschevnikovi contained more spermatozoa than reported from $A C$ indica. A c cerana queens mated in Pakistan had 2.7 and 3.8 mio spermatozoa (Ruttner and Maul, 1969, 1983). Queens of European A mellifera races have about 360 ovarioles and about 5 mio spermatozoa in the spermatheca, which also has a bigger volume.
The main features of mating behavior in A koschevnikovi show similar patterns as the other cavity nesting species: mating occurs outside the hive; the queen performs 1 or 2 mating flights; sperm is transferred into the oviducts; and queens return with a mating sign. Within the next hours only a small percentage of the spermatozoa received reach the spermatheca.

Within this framework, A koschevnikovi and $A$ cerana have more similarities to each other than to A mellifera:

(i) weight relationship of queens, drones and workers;

(ii) number of spermatozoa of drones;

(iii) morphology of the mating sign (no chitin plates);

(iv) number of ovarioles in queens.

This listing further underlines the close relationship between $A$ koschevnikovi and $A$ cerana, which has previously been established by a number of synapomorphic characteristics, such as the pore in the capping of the drone brood cell (Otis, 1990), the fanning position of the worker and the same species of a parasitic mite (Varroa jacobsoni).

\section{ACKNOWLEDGMENTS}

We dedicate this article to Friedrich Ruttner on occasion of his 80th birthday. More than $30 \mathrm{yr}$ ago, he pioneered research into the reproduction of Asian honeybee species. 
Deutsche Version

\title{
Hochzeitsflug, Spermaübertragung und Mehrfachpaarung bei Apis koschevnikovi (Buttel-Reepen, 1906)
}

\begin{abstract}
Zusammenfassung - Königinnen und Drohnen von $A$ koschevnikovi verlassen ihr Volk zur Paarung. Die Abflüge der Drohnen erfolgten zwischen 16.45 und $18.30 \mathrm{Uhr}$, die Flugdauer schwankte zwischen weniger als $1 \mathrm{~min}$ bis $27 \mathrm{~min} .73 \%$ der Ausflüge, die länger als $10 \mathrm{~min}$ dauerten, fanden am Ende der Flugperiode zwischen 17.45 und 18.30 Uhr statt. Die Abflüge der Königinnen erfolgten zwischen 17.00 und 18.15 Uhr. Hier erfolgten die Abflüge zur Paarung in einer kurzen Zeitspanne zwischen 17.53 und 18.03 Uhr. Die Flugdauer betrug bei Rückkehr mit Begattungszeichen im Mittel 19 $\pm 5 \mathrm{~min}$. Die Ovidukte der heimkehrenden Königinnen enthielten zwischen 12 und 21 Mio Spermatozoen. Da ein Drohn 1,7 $\pm 0,16$ Mio Spermatozoen hat, wurden die Königinnen von 7 bis 12 Drohnen auf einem Flug gepaart. Die Begattungszeichen ähneln in der Form denen von $A$ cerana, Chitinspangen wie bei $A$ mellifera sind nicht vorhanden. Eierlegende Königinnen hatten zwischen 1,5 und 3 Mio Spermatozoen in der Spermatheka. Beim Vergleich des Reproduktionsverhaltens ergaben sich für $A$ koschevnikovi und $A$ cerana sehr viele Ähnlichkeiten.
\end{abstract}

Paarungsverhalten / Anzahl der Spermatozoen / Mehrfachpaarung / A koschevnikovi

\section{EINLEITUNG}

Die höhlenbrütende 'Rote Biene von Sabah' wurde erst 1988 auf Borneo wiederentdeckt (Tingek et al, 1988; Mathew and Mathew, 1990). Maa hatte diese Bienenart 1956 beschrieben und gab ihr den Namen Apis vechti. 1989 publizierten Ruttner et al, daß die erste Beschreibung 1906 durch ButtelReepen erfolgte. Er nannte diese Biene Apis koschevnikovi und dies ist der gültige Name.

Die 'Rote Biene' A koschevnikovi sieht äußerlich der sympatrischen Honigbiene $A$ cerana sehr ähnlich, ist aber größer und auffällig rötlich. Es handelt sich um eine getrennte Art. Die reproduktive Isolation zu den drei anderen in Sabah sympatrischen Arten der Honigbiene, Apis andreniformis, $A$ cerana und $A$ dorsata, wurde durch unterschiedliche Drohnenflugżeiten (Koeniger et al, 1988) und den unterschiedlichen Bau des männlichen Begattungsorgans (Tingek et al, 1988; Koeniger et al, 1991) gezeigt. Außer- dem bildete $A$ koschevnikovi nach morphometrischen Analysen ein eigenes Cluster (Rinderer et al, 1989; Ruttner et al, 1989).

Das Paarungsverhalten der europäischen Biene A mellifera lag schon seit Jahrhunderten im Interesse der Wissenschaft (Janscha 1775; Übersicht von Ruttner, 1985). Erst kürzlich wurden auch einige Einzelheiten über das Paarungsverhalten von A cerana bekannt (Ruttner und Maul, 1969, 1983; Ruttner et al, 1972, 1973; Ruttner, 1973; Woyke, 1975; Punchihewa et al, 1990; Punchihewa, 1992; Yoshida and Saito, 1990; Yoshida et al, 1994; Fujiwara et al, 1994). Von A koschevnikovi fehlen dagegen noch wesentliche Kenntnisse zur Paarungsbiologie. Wir berichten nun über Ausflüge von Königinnen und Drohnen, über ihr Gewicht und die Anzahl der Spermatozoen, aus denen man auf die Spermaübertragung und den Grad der Mehrfachpaarung schließen kann. 


\section{MATERIAL UND METHODE}

Alle Versuche wurden in der Landwirtschaftlichen Station in Tenom, Sabah, Ost-Malaysia durchgeführt. Dort werden $A$ koschevnikovi Völker seit einigen Jahren in Magazinen mit beweglichen Waben gehalten. Die Versuchsvölker wurden in der Station und ihrer Umgebung aus der natürlichen Population gefangen.

Um das Körpergewicht von Arbeiterinnen, Königinnen und Drohnen zu bestimmen, wurden sie einzeln in kleine Plastikröhrchen gesteckt und auf einer digitalen Waage gemessen (Genauigkeit $0,1 \mathrm{mg}$ ).

Verdeckelte Weiselzellen wurden in kleine weisellose Völker (2 000-3 000 Bienen) an einer Wabe befestigt. Täglich wurden die Völker kontrolliert und die frisch geschlüpften Königinnen wurden mit einem numerierten Zeichenplättchen markiert.

Am Flugloch wurde ein kleiner Vorbau angebracht, dessen Oberseite aus Glas und dessen Vorderseite mit einem Absperrgitter $(4,0 \mathrm{~mm}$ Abstand) versehen war (Ruttner, 1954). Die Arbeiterinnen konnten durch das Gitter schlüpfen, nicht aber die größeren Königinnen. Sie wurden, sowie sie unter dem Glas im Vorbau erschienen, durch kurzzeitige Entfernung des Glasdaches freigelassen. Bei ihrer Rückkehr landeten die Königinnen außen an dem Vorbau und konnten untersucht werden.

Für die Bestimmung der Flugzeit haben wir die Abflüge der Drohnen und Königinnen protokolliert. Für die Bestimmung der Flugdauer wurden die Drohnen individuell mit verschiedenen Farben und Mustern markiert (Edding lac marker). Bei 3 Völkern wurden An- und Abflüge der einzelnen Drohnen beobachtet.

Die Anzahl der Spermatozoen wurde nach den Beschreibungen von Mackensen und Ruttner (1976) durchgeführt. Der Grad der Verdünnung des Spermas lag je nach Spermamenge zwischen 5 und $20 \mathrm{ml}$.

\section{ERGEBNISSE}

\section{Gewichte}

Zwei eierlegende Königinnen wogen 179 $\mathrm{mg}$ und $171 \mathrm{mg}, 3$ vom Hochzeitsflug zurückkehrende Königinnen 145 mg, 150 $\mathrm{mg}$ und $153 \mathrm{mg}$ (Tabelle II). Drohnen hatten ein Durchschnittsgewicht von 101,2 $\pm 4,1$ $\mathrm{mg}(n=20)$ und Arbeiterinnen 72,1 $\pm 7,7$ $\mathrm{mg}(n=20)$. Die Anzahl der Ovariolen der Königinnen betrug 137,4 $\pm 3,9(n=5)$, der Durchmesser ihrer Spermatheken $d=0,74$ $\pm 0,03 \mathrm{~mm}$. Das entspricht einem Volumen von etwa $0,25 \mathrm{~mm}^{3}$.

\section{Flug der Königinnen}

Die Flugaktivität der Königinnen begann um 17.10 und endete um 18.29 Uhr, der letzte Abflug erfolgte um 18.13 Uhr (Abb 1). Der Median für alle Abflüge lag bei $17.46 \mathrm{Uhr} \pm$ $15 \mathrm{~min}$. Alle Flüge vor 17.53 Uhr waren Orientierungsflüge (Rückkehr ohne Begattungszeichen). Ihre Dauer betrug 1,9 \pm 1,4 $\min (n=30$, Minimum $1 \mathrm{~min}$, Maximum 7 min; Abb 2). Zu Hochzeitsflügen (Rückkehr mit Begattungszeichen (Abb 3 ) starteten die Königinnen zwischen 17.53 und 18.03 Uhr. Die Dauer betrug 19,0 5 min ( $n=9$, Minimum 12 min, Maximum 27 min, Abb 2). Der Median für die Abflugzeit der Hochzeitsflüge lag bei 17.59 Uhr $\pm 3,6 \mathrm{~min}$.

Sechs Königinnen unternahmen im Alter von 4 und 5 Tagen ihre ersten Orientierungs-, im Alter von 5 und 6 Tagen ihre Hochzeitsflüge. Sie machten im Durchschnitt 3,5 Orientierungsflüge, da auch vor dem 2 Hochzeitsflug wieder 1-2 Orientierungsflüge stattfanden. Eine Königin begann ihre Orientierungsflüge erst im Alter von 7 Tagen und kehrte nach dem 10.Ausflug im Alter von 11 Tagen nicht zurück.

\section{Drohnenflüge}

Die Drohnenflüge begannen um 16.45 Uhr am Nachmittag, die letzten Abflüge erfolgten gegen 18.30 Uhr. Die meisten Abflüge $(85 \%)$ lagen zwischen 17.15 und $18.00 \mathrm{Uhr}$ (Abb 1). Die Flugdauer differierte von weni- 
ger als $1 \mathrm{~min}$ bis $27 \mathrm{~min}$ (Abb 2). Sie wurden nach Drescher (1968) und in Anlehnung an das Verhalten der Königinnen in Orientierungsflüge (bis zu $10 \mathrm{~min}$ ) und Paarungsflüge (länger als $10 \mathrm{~min}$ ) unterteilt. $93 \%$ der Paarungsflüge wurden zwischen 17.30 und 18.30 Uhr unternommen, während kurze Flüge sich über die gesamte Flugzeit erstreckten. Der Median für alle Abflüge lag bei 17.45 Uhr $\pm 11 \mathrm{~min}$, für die Abflüge zur Paarung bei $17.53 \mathrm{Uhr} \pm 10 \mathrm{~min}$. Die durchschnittliche Flugdauer aller Flüge betrug $12,3 \pm 8 \min (n=66)$, ohne Berücksichtigung der Orientierungsflüge betrug sie 16,0 $\pm 6 \min (n=48)$.

\section{Das Begattungszeichen}

Die Begattungszeichen bestanden aus einer dünnen durchsichtigen Hülle, in die Mucus aus den Anhangsdrüsen eingepreßt und als ein in sich gefalteter Faden zu erkennen war. An den Seiten aufgelagert ist das orangene Sekret der Cornualdrüsen (Koeniger et al, 1990), das am distalen Ende überlappt. Im Gegensatz zu A mellifera sind keine Chitinspangen vorhanden $(A b b 3,4)$.

\section{Anzahl der Spermatozoen}

\section{Drohnen}

Die Vesicula seminales der Drohnen enthielten 1,7 0,16 Mio Spermatozoen ( $n=$ 20). Im Ejakulat von 5 künstlich zur Eversion gebrachten Drohnen befanden sich zwischen 1,1 und 1,3 Mio.

\section{Königinnen}

Nach dem ersten erfolgreichen Hochzeitsflug enthielten die Ovidukte von 3 Königinnen 16,3 Mio, 19,0 Mio und 21,1 Mio Spermatozoen. Eine Königin hatte nach ihrem
2. Hochzeitsflug 2 Mio Spermatozoen in der Spermatheka und 12 Mio in den Ovidukten.

Die Spermatheka einer Königin, die nach dem 2. Hochzeitsflug mit der Eilage begann, enthielt 3,0 Mio Spermatozoen. Bei eierlegenden Königinnen unbekannten Alters enthielten sie 1,4 Mio, 1,6 Mio und 2,5 Mio.

\section{Anzahl der Paarungen von 4 Königinnen}

Drei Königinnen hatten nach dem ersten Hochzeitsflug zwischen 16,3 Mio und 21,1 Mio Spermatozoen in den Ovidukten. Das entspricht der Spermamenge von 10 bis 12 Drohnen. Eine Königin hatte nach ihrem 2. Hochzeitsflug 12 Mio Spermatozoen in den Ovidukten, 2 Mio befanden sich in der Spermatheka. Auch diese Königin muß sich insgesamt mit mehr als 10 Drohnen gepaart haben.

\section{DISKUSSION}

Wie bei anderen Arten der Honigbienen verließen Königinnen und Drohnen ihr Volk zur Paarung. Der Drohnenflug begann 15 min früher und dauerte auch einige Minuten länger als der Königinnenflug. Die Medianzeit stimmt aber bei beiden fast überein (17.45 h und 17.46 h). Zwei Arten Flüge wurden nach der Flugdauer klassifiziert: Orientierungs- bzw Reinigungsflüge (kürzer als 10 min) und Paarungsflüge (länger als $10 \mathrm{~min}$ ) wie von Ruttner (1985) für Königinnen und von Drescher (1968) für Drohnen bereits für $A$ mellifera beschrieben wurde. Die Paarungsflüge der Drohnen begannen später und erfolgten in einer kürzeren Zeitspanne von etwa 1 Stunde als die Orientierungsflüge, die sich über die gesamte Flugzeit von $13 / 4$ Stunden erstreckten. Die Königinnen starteten zwischen 17.53 und 18.03 Uhr zum Hochzeitsflug. Diese kurze Zeitspanne mag auch durch die geringe Zahl 
(9) der von uns beobachteten Hochzeitsflüge bedingt sein.

Die Dauer der Hochzeitsflüge der Königinnen schwankte zwischen 12 und $27 \mathrm{~min}$. Das ist eine ähnliche Dauer wie für $A \mathrm{~m}$ carnica (Ruttner, 1985) und auch für $A$ cerana Königinnen in Indien (Adlakha, 1971: 15-29 min; Woyke, 1975: 27 min im Durchschnitt) gemessen wurde. In Sri Lanka war die Paarungszeit der $A c$ indica mit 7 bis $12 \mathrm{mir}$ ( $n$ $=28$; Punchihewa, 1992) wesentlich kürzer. Diese Differenz kann durch die Anzahl der Drohnen in der Umgebung entstanden sein. Aber auch andere Umwelteinflüsse mögen einen Einfluß auf die Flugzeit haben.

Die Dauer der Orientierungsflüge der Drohnen war etwa genauso lang wie sie von $A C$ indica (Punchihewa, 1992) und $A$ mellifera (Drescher, 1968; Berg, 1992) beschrieben wurden. Die Paarungsflüge waren mit 16 min dagegen kürzer als bei $A$ mellifera (20 bis $30 \mathrm{~min}$ ) und länger als bei A c indica (12 min) in Sri Lanka.

Wie bei den anderen höhlenbrütenden Arten der Honigbienen gelangten bei den Königinnen von $A$ koschevnikovi während des Hochzeitsfluges viel mehr Spermatozoen in die Ovidukte als in der Spermatheka gespeichert werden können. Mehr als $80 \%$ werden wieder ausgeschieden. Im allgemeinen scheinen sich die meisten Königinnen der 3 höhlenbrütenden Arten mit 10 bis 12 Drohnen zu paaren (A c indica: Woyke, 1975; Punchihewa, 1992 / europäische Rassen von A mellifera: Woyke, 1960; Cornuet et al, 1986; Haberl und Moritz, 1994). Für Bienen in Süd-Amerika wurden im Mittel 17 Paarungen berechnet (Adams et al, 1977). Bei einer $A$ cerana Königin aus Pakistan, die nach Ruttner (1988) zu der nördlichen Rasse A c cerana gehört, wurden sogar 30 Paarungen geschätzt (Ruttner et al, 1973).

Die Anzahl der Spermatozoen in den Vesicula seminales der Drohnen sind mit 1,7 Mio bei $A$ koschevnikovi und 1 Mio bei $A c$ indica signifikant unterschiedlich. A mellifera hat die 6 bzw. 10-fache Menge. $A$ koschevnikovi Drohnen sind halb so schwer wie $A$ mellifera Drohnen, sie haben aber nur $17 \%$ der Spermienzahlen. A $c$ indica Drohnen sind um $17 \%$ leichter als $A$ koschevnikovi Drohnen und haben $41 \%$ weniger Spermatozoen. Ein A c cerana Drohn ist größer als ein $A c$ indica Drohn (Ruttner, 1988) und hat mit 1,5 Mio auch mehr Spermatozoen (Ruttner und Maul, 1969, 1983). Dagegen haben kleine Drohnen von $A$ m carnica, die aus Arbeiterinnenzellen geschlüpft sind, fast genausoviele Spermatozoen wie normal große Drohnen (Berg, 1992). A florea Drohnen wiederum, die genausoviel wiegen wie $A c$ indica Drohnen (Koeniger et al, 1994), haben nur $40 \%$ der Spermamenge. Demnach folgt die Anzahl der Spermatozoen zwar der allgemeinen Regel, daß größere Männchen auch mehr Spermatozoen haben, aber zusätzlich ist eine artspezifische Komponente vorhanden. Bei A florea zum Beispiel dürfte der unterschiedliche Mechanismus der Spermaübertragung direkt in die Spermatheka einen Einfluß haben (Koeniger et al, 1990).

Die Morphologie des Begattungszeichens ist ganz anders als bei $A$ mellifera: es gibt keine Chitinspangen. Außerdem ist es länglich gebaut. An beiden Seiten liegt je eine Schicht des Cornualsekrets (Koeniger et al, 1990), das am distalen Ende verschmilzt. Es hat große Ähnlichkeit mit dem von A cerana (Ruttner et al, 1973; Woyke, 1975; Punchihewa, 1992).

A koschevnikovi ist etwas größer als die sympatrische A cerana. Sowohl Arbeiterinnen als auch die Geschlechtstiere wiegen etwa um 1/5 mehr. Aber die Gewichtsrelation von Arbeiterinnen zu Geschlechtstieren ist bei $A$ cerana und $A$ koschevnikovi sehr ähnlich. Bei Vergleich von Arbeiterin / Drohn / Königin beträgt es 1/1,4/2,1. Das heißt Drohnen sind etwas schwerer und Königinnen mehr als doppelt so schwer wie Arbeiterinnen. Bei $A$ mellifera ist es deutlich verschieden mit 1/2,4 / 2,3 (Koeniger et 
al, 1994), die Drohnen sind hier sogar noch schwerer als die Königinnen.

Die Zahl der Ovariolen war mit 137,4 \pm $3,9(n=5)$ ähnlich wie in A cerana, obwohl die Königinnen von $A$ koschevnikovi $25 \mathrm{mg}$ schwerer waren. Wiederum ist diese Eigenschaft sehr unterschiedlich zu $A$ mellifera (360 Ovariolen). Die Spermatheken hatten etwa das gleiche Volumen wie $A c$ indica (Woyke, 1975), aber die beiden frisch gepaarten $A$ koschevnikovi Königinnen hatten mit 2 Mio (zusätzlich $12 \mathrm{Mio}$ in den Ovidukten) und 3 Mio etwa doppelt so viele Spermatozoen in der Spermatheka. Bei A c cerana wurden zwischen 2,5 und 3,5 Mio Spermatozoen gezählt (Ruttner und Maul, 1983).

Viele Befunde bei der Reproduktion von A koschevnikovi stimmen im Prinzip mit den beiden anderen höhlenbrütenden Arten $A$ mellifera und $A$ cerana überein: Die Paarung erfolgt entfernt vom Volk, Königinnen unternehmen 1 bis 2 Hochzeitsflüge, das Sperma wird zunächst in die Ovidukte übertragen und die Königin kehrt mit einem Begattungszeichen zurück. Erst in den folgenden Stunden erfolgt die Füllung der Spermatheka, wobei weniger als $20 \%$ der Spermatozoen gespeichert werden. Innerhalb dieses Rahmens aber gibt es viele Eigenschaften, die $A$ koschevnikovi sehr nah zu $A$ cerana stellen:

1) Die Gewichtsrelation von Arbeiterin, Drohn und Königin.

2) Die Anzahl der Spermatozoen bei Drohnen.

3) Die Morphologie des Begattungszeichens (keine Chitinspangen).

4) Die Anzahl der Ovariolen der Königin.

Diese Aufzählung unterstreicht die enge Verwandtschaft zwischen A koschevnikovi und $A$ cerana, die bereits mit anderen synapomorphen Eigenschaften gezeigt wurde, wie die Pore im Deckel der Drohnenbrutzellen (Otis, 1990), die Stellung beim Fächeln und der gleiche Brutparasit Varroa jacobsoni.

\section{DANKSAGUNG}

Diese Arbeit ist Friedrich Ruttner zu seinem 80. Geburgstag gewidmet, der als Pionier vor mehr als 30 Jahren mit der Erforschung der Reproduktion von asiatischen Honigbienenarten begann.

Résumé - Vol de fécondation, nombre de spermatozoïdes, transfert du sperme et degré de polyandrie chez Apis koschevnikovi (Buttel-Reepen, 1906). Le comportement d'accouplement d'Apis cerana et d'Apis mellifera a été étudié par de nombreux auteurs. Pour A koschevnikovi, en revanche, on ne dispose pas des connaissances de base. Nous présentons dans cette étude des données sur le poids des mâles et des reines et sur leur vol de fécondation. D'après le nombre de spermatozoïdes des mâles et celui des reines récemment accouplées, on peut déduire le nombre d'accouplements et le transfert de sperme. Le poids de 2 reines pondeuses était de 171 et $179 \mathrm{mg}$, celui de 3 jeunes reines compris entre 145 et $153 \mathrm{mg}$. Les mâles $(n=20)$ pesaient entre 101,2 $\pm 4,1$ mg et les ouvrières ( $n=2$, tableau II) $72,1 \pm$ $7,7 \mathrm{mg}$. Les individus des 3 castes d' $A$ koschevnikovi sont donc plus lourds que ceux de l'espèce sympatrique $A c$ indica. Le rapport pondéral ouvrière / mâle / reine est très semblable chez les 2 espèces: $1 /$ 1,4 / 2,1. Chez $A$ mellifera il est nettement différent: 1 / 2,4 / 2,3. Le nombre d'ovarioles $137,4 \pm 3,9(n=5)$ est proche de celui d' $A$ cerana mais, là encore, très différent de celui d'A mellifera (340 ovarioles). Les mâles s'envolaient entre 16 h 45 et 18 h 30 (fig 1) ; la durée du vol allait de moins de $1 \mathrm{~min}$ jusqu'à 27 min (fig 2). 73\% des vols supérieurs à $10 \mathrm{~min}$ (vols de fécondation) démarraient entre $17 \mathrm{~h} 45$ et $18 \mathrm{~h} 15$ (fig 1 ). Les reines quittaient la ruche entre $17 \mathrm{~h} 00$ et $18 \mathrm{~h} 15$ (fig 1). Les vols des reines duraient en moyenne 1,9 $\pm 1,4$ min pour les vols d'orientation (pas de signe de fécondation, 
$n=30)$ et $19 \pm 5 \min (n=9$, fig 2$)$ pour les vols de fécondation (présence d'un signe de fécondation, fig 3). Les départs pour les vols de fécondation avaient lieu dans un laps de temps court entre $17 \mathrm{~h} 53$ et $18 \mathrm{~h} 00$. Les signes de fécondation ressemblaient tout à fait à ceux d'A cerana (fig 4). Ils consistaient en un épais fil de mucus blanc replié en zig-zag dans une fine enveloppe transparente. À sa surface était attachée la sécrétion orange des cornules. Contrairement à $A$ mellifera il n'y a pas ici de plaques chitineuses. Les vésicules séminales des mâles renfermaient $1,7 \pm 0,16$ million de spermatozoïdes $(n=20)$, les oviductes des 3 reines rentrant de leur premier vol de fécondation en contenaient 16,3, 19,0 et 21,1 millions. L'une des reines, disséquée après 2 vols de fécondation fructueux, avait 12 millions de spermatozoïdes dans les oviductes et 2 millions dans la spermathèque. Comme chez les autres espèces qui nidifient dans des cavités, le transfert de sperme a lieu chez $A$ koschevnikovi d'abord dans les oviductes. Ce n'est qu'ensuite qu'un certain pourcentage de spermatozoïdes atteint la spermathèque. Le nombre d'accouplements (avec 10 à 12 mâles) est lui aussi semblable aux 2 autres espèces. Le nombre de spermatozoïdes dans les vésicules séminales des mâles est significativement différent chez $A$ koschevnikovi $(1,7 \pm 0,16$ millions) et $A c$ indica ( $1 \pm 0,1$ million). II s'agit de savoir si le nombre de spermatozoïdes dépend de la taille du mâle ou bien est spécifique à l'espèce. Les petits mâles d' $A C$ indica ont également le plus petit nombre de spermatozoïdes. Les mâles d'A florea, qui pèsent autant que ceux d'A $c$ indica, n'ont que $40 \%$ de spermatozoïdes. En comparant les données des diverses races et espèces, on s'aperçoit que le nombre de spermatozoïdes suit la règle générale selon laquelle les mâles les plus gros ont le plus grand nombre de spermatozoïdes, mais qu'il y a en plus une composante liée à l'espèce. Chez $A$ florea par exemple le transfert direct du sperme dans la spermathèque doit avoir une influence. Les données concernant la reproduction d' $A$ koschevnikovi concordent dans l'ensemble avec celles des autres espèces nidifiant dans des cavités : l'accouplement a lieu loin de la colonie, les reines accomplissent 1 ou 2 vols de fécondation, le sperme est transféré dans les oviductes et la reine rentre avec un signe de fécondation. Ce n'est que dans les premières heures qui suivent que la spermathèque se remplit. Mais dans ce schéma, $A$ koschevnikovi ressemble plus à $A$ cerana qu'à $A$ mellifera : i) par la relation pondérale ouvrière/ mâle/reine, ii) par le nombre de spermatozoïdes des mâles, iii) par la morphologie du signe de fécondation (pas de plaques chitineuses), et iv) par le nombre d'ovarioles de la reine. Cette liste souligne l'étroite parenté entre $A$ koschevnikovi et $A$ cerana, que d'autres caractères synapomorphiques ont déjà montrée: le pore dans l'opercule des cellules de mâles, la position lorsque les abeilles battent le rappel et le même parasite du couvain Varroa jacobsoni Oud.

vol de fécondation / spermatozoïde / polyandrie / A koschevnikovi 
Table I. Weight (mg) of queens, drones and workers in the 3 cavity nesting Apis species.

Tabelle I. Gewicht von Königin, Drohn und Arbeiterin bei den drei höhlenbrütenden Apis Arten.

Species

A cerana indica

A koschevnikovi

A mellifera ligustica

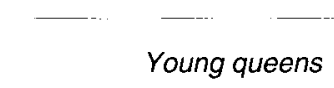

$122 \pm 13^{*}$

$145-153$

$198 \pm 13^{\star *}$

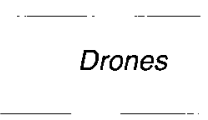

$83 \pm 5^{\star *}$

$101 \pm 4$

$211 \pm 18^{* *}$

\section{Workers}

$60 \pm 9^{* *}$

$72 \pm 8$

$86 \pm 11^{* *}$

* Lap et al, 1992; ** Koeniger et al, 1994.

Table II. Number of spermatozoa in millions of drones and in queens in the 3 cavity nesting Apis species.

Tabelle II. Anzahl der Spermatozoen von Drohnen und in Königinnen in Millionen bei den drei höhlenbrütenden Apis Arten

\begin{tabular}{lccc} 
Species & $\begin{array}{l}\text { Drone } \\
\text { vesicula seminalis }\end{array}$ & $\begin{array}{l}\text { Queen } \\
\text { oviducts }\end{array}$ & $\begin{array}{l}\text { Queen } \\
\text { spermatheca }\end{array}$ \\
\hline A cindica** & $1.0 \pm 0.1$ & $12 \pm 1.0$ & $1.3 \pm 0.1$ \\
A c cerana & 1.5 & - & $2.5-3.5$ \\
Apis koschevnikovi & $1.7 \pm 0.16$ & $12-21$ & $2-3$ \\
A m carnica & $10.3 \pm 0.35$ & $75 \pm 32$ & $4.7 \pm 0.5$ \\
\hline
\end{tabular}

* Ruttner and Maul, 1969; Ruttner et al, 1973; ** Punchihewa 1992; Woyke 1975; *** Calculated after Woyke 1960, 1966. 

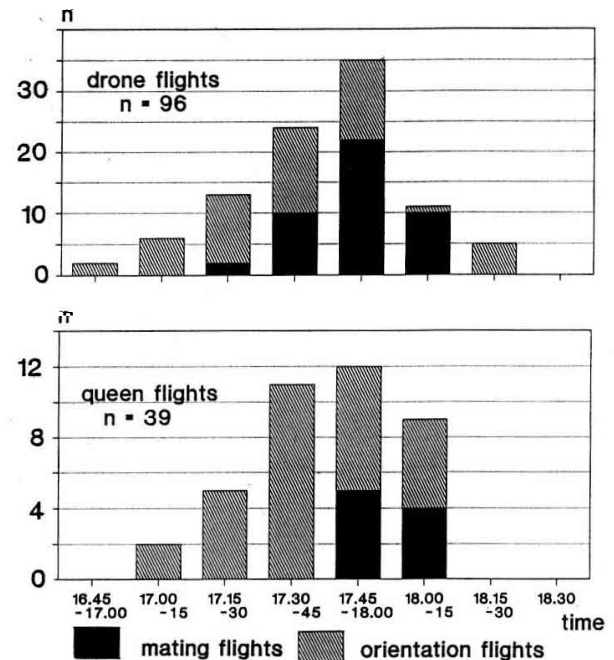

Fig 1. Time of departures for orientation and mating flights of queens and drones.

$A b b$ 1. Abflugszeit der Orientierungs- und Paarungsflüge von Königinnen und Drohnen. Abszisse: Abflugszeit / Ordinate: Anzahl der Flüge.

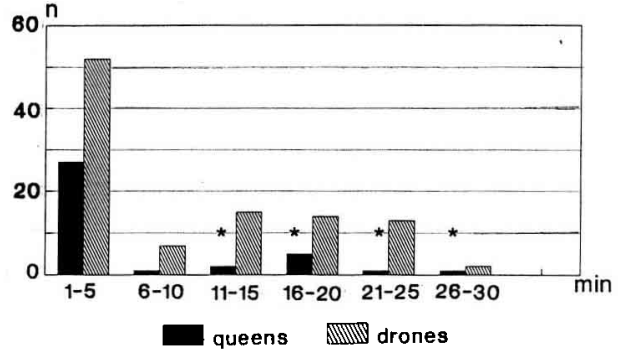

* queen returned with mating sign

Fig 2. Duration of flights of queens and drones (min).

Abb 2. Dauer der Ausflüge von Königinnen und Drohnen in Minuten.

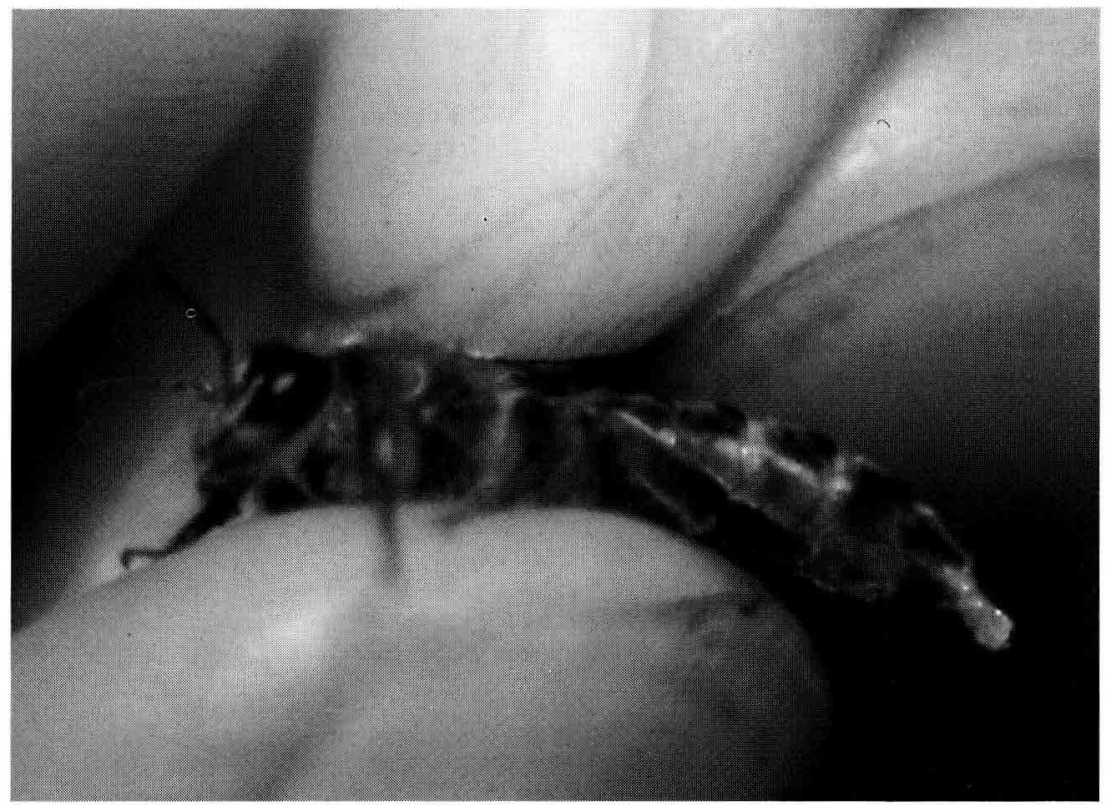

Fig 3. A koschevnikovi queen with mating sign.

$A b b$ 3. Eine A koschevnikovi Königin mit Begattungszeichen. 


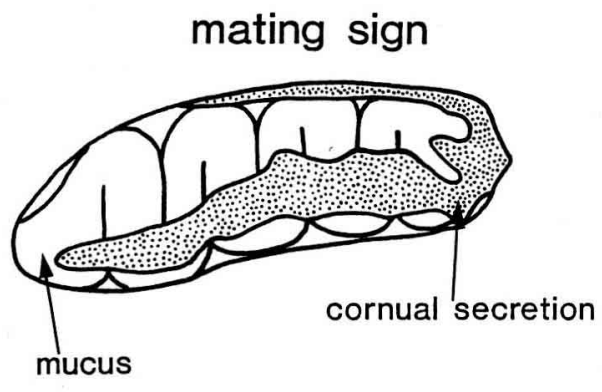

Fig 4. Mating sign of $A$ koschevnikovi.

Abb 4. Begattungszeichen von A koschevnikovi.

\section{REFERENCES}

Adams J, Rothmann ED, Kerr WE, Paulino ZL (1977) Estimation of sex alleles and queen matings from diploid male frequencies in a population of Apis mellifera. Genetics 86, 583596

Adlakha RL (1971) Pecularities of mating of honeybee queens of Apis mellifera and Apis cerana and beginning of oviposition. Proc 23 Int Beekeep Congr, Bucharest Apimondia, 434-439

Berg S (1992) Paarungserfolg von großen und kleinen Drohnen. Dissertation, Universität Frankfurt, Germany

Cornuet JM, Daoudi A, Chevalet C (1986) Genetic pollution and number of matings in a black honey bee (Apis mellifera mellifera) population. Theor Appl Genet 73, 223-227

Drescher W (1968) Die Flugaktivität von Drohnen der Rasse Apis mellifera carnica $L$ und Apis mellifera ligustica in Abhängigkeit von Lebensalter und Witterung. $Z$ Bienenforsch 9 , 390-409

Fujiwara S, Miura $H$, Kumagai $T$, Sawaguchi $T$, Naya $W$, Goto KT, Asanuma $H$, Suzuki $K$ (1994) Drone congregation of Apis cerana japonica (Radozgkowski 1877) above large trees. Apidologie 25 (in press)

Haberl, Moritz RFA (1994) Estimation of intracoIonial worker relationship in a honey bee colony (A mellifera L). Insectes Soc (in press)

Janscha A (1775) Vollständige Lehre von der Bienenzucht. G Münzberg, Wien
Koeniger G, Wissel M, Herth W (1990) Cornual secretion on the endophallus of the honeybee drone (A mellifera). Apidologie 21, 186191

Koeniger G, Koeniger N, Mardan M, Otis G, Wongsiri $S$ (1991) Comparative anatomy of male genital organs in the genus Apis. Apidologie 21, 539-552

Koeniger G, Koeniger N, Mardan M, Wongsiri S (1994) Variance in weight of sexuals and workers within and between 4 Apis species. In: Asian apiculture (LJ Connor, T Rinderer, A Sylvester, S Wongisir, eds) Proc 1st Int Conf on Asian honey bees and mites, Cheshire USA, Wicwas Press (in press)

Koeniger N, Koeniger G, Tingek S, Mardan M, Rinderer TE (1988) Reproductive isolation by different time of drone flight between Apis cerana Fabricius, 1793 and Apis vechti (Maa, 1953). Apidologie 19, 103-106

Koeniger N, Koeniger G, Wongsiri S (1989) Mating and sperm transfer in Apis florea. Apidologie 20, 414-418

Lap PV, Chinh PH, Chinh TX (1992) Some biological characteristics of $A$ cerana queen bees in Vietnam. Abstracts Int. Conf. on Asian honey bees and bee mites. Bangkok, Thailand

Maa TC (1956) An inquiry into the systematics of the tribus Apidini or honeybees (Hymenoptera). Treubia, 525-640

Mackensen O, Ruttner F (1976) The insemination procedure. In: The instrumental insemination of honeybee queens (F Ruttner, ed). Bucharest Apimondia 69-86

Mathew KP, Mathew S (1990) Further observations on characters and behaviour of Apis koschevnikovi, the "red honeybee" of Sabah. Bee World 71, 61-66

Otis $G$ (1990) A review of the diversity of species. In: Diversity in the genus Apis (DR Smith, ed) Westview Press, Oxford, 29-49

Punchihewa RWK, Koeniger N, Koeniger G (1990) Congregation of Apis cerana indica (Fabricius 1798) drones in the canopy of trees in Sri Lanka. Apidologie 21, 201-208

Punchihewa RWK (1992) Beobachtungen und Versuche zum Paarungsverhalten von Apis cerana indica in Sri Lanka. Dissertation, Universität Frankfurt, Germany 
Rinderer TE, Koeniger N, Tingek S, Mardan M, Koeniger G (1989) A morphological comparison of the cavit dwelling honeybees of Borneo Apis koschevnikovi (Buttel-Reepen 1906) and Apis cerana (Fabricius 1794). Apidologie 20, 405-411

Ruttner F (1954) Mehrfache Begattung der Bienenkönigin. Zool Anz 153, 99-105

Ruttner F (1973) Drohnen von A cerana Fabr auf einem Drohnensammelplatz. Apidologie 4, 41-44

Ruttner F (1985) Reproductive behaviour in honeybees. In: Fortschritte der Zoologie, Bd 31 (Hölldobler/Lindauer, eds) "Experimental behavioral Ecology", G Fischer Verlag, Stuttgart, 225-236

Ruttner F (1988) Biogeography and taxonomy of honeybees. Springer Verlag, Berlin

Ruttner F, Maul V (1969) The cause of hybridization barrier between Apis mellifera $\mathrm{L}$ and Apis cerana Fabr. XXII Int Beekeep Congr, Bucharest, Apimondia, 561

Ruttner F, Maul V (1983) Experimental analysis of reproductive interspecies isolation of Apis mellifera $L$ and Apis cerana Fabr. Apidologie 14, 309-327

Ruttner F, Woyke J, Koeniger N (1972) Reproduction in Apis cerana. I. Mating behavior. $J$ Apic Res 11, 141-146

Ruttner F, Woyke J, Koeniger N (1973) Reproduction in Apis cerana. 2. Reproductive organs and natural insemination. J Apic Res 12, 2134
Ruttner F, Kauhausen D, Koeniger N (1989) Position of the red honey bee, Apis koschevnikovi (Buttel-Reepen 1906), within the genus Apis. Apidologie 29, 295-404

Tingek S, Mardan M, Rinderer TE, Koeniger N, Koeniger G (1988) Rediscovery of Apis vechti (Maa 1953): the Saban honeybee. Apidologie 19, 97-102

Woyke $J(1960)$ Natural and artificial insemination of queen honeybees. Pszcz Zesz Nauk 4, 183-275

Woyke J (1966) Wovon hängt die Zahl der Spermien in der Samenblase der auf natürlichem Wege begatteten Königinnen ab? Z Bienenforsch 8, 2366-248

Woyke J (1975) Natural and instrumental insemination of Apis cerana indica in India. J Apic Res 19, 153-159

Yoshida T, Saito J (1990) Mating success of both native $A$ cerana japonica and introduced $A$ mellifera in sympatric conditions. In: Social insects and the environment, proceedings of the 11th internat Congr of IUSSI (GK Veeresh, B Mallik, CA Virakthamath, eds) Oxd \& IBH Publishing Co LTD, Bombay Delhi, 101$102 \mathrm{p}$

Yoshida T, Saito J, Kajigaya N (1994) The mating flight times of native Apis cerana japonica (Radoszkowski 1877) and introduced Apis mellifera Linnaeus 1758. Apidologie 25 (in press) 